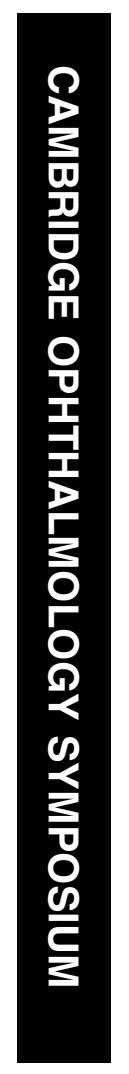

\title{
Transplantation prospects for the inner retina
}

\begin{abstract}
Transplantation of stem or progenitor cells is an attractive new approach for treating neurodegenerative conditions of the central nervous system, which aims to protect or replace neurons and improve function. Proof of principle has already been shown in the retina that photoreceptors may be replaced by transplantation of neural progenitor cells. However, the task of retinal ganglion cell replacement is much more complex, as new cells will need to establish complex connections within the retina and also extend axons to precise targets in the brain. Although progress has been made in this field, it is likely that neuroprotective clinical applications will be established more quickly. Our laboratory has focused on the intraocular transplantation of cells to treat inner retinal disease, either by neuronal replacement or neuroprotection of existing cells. We have investigated the efficacy and effects of transplanting a variety of cell types, including human Müller stem cells (MIO-M1), oligodendrocyte precursor cells (OPCs), and bone marrow-derived mesenchymal stromal cells (MSCs) in a rat model of experimentally induced glaucoma. We also have developed and characterized a novel in vitro organotypic retinal explant culture system for exploring the methods of enhancing the efficacy of cell transplantation for the inner retina. In this review, we discuss the potentially beneficial effects of intraocular cell injections, identify current shortcomings of retinal stem cell therapy, and suggest directions for future research.

Eye (2009) 23, 1980-1984; doi:10.1038/eye.2008.376; published online 19 December 2008
\end{abstract}

Keywords: stem cell; transplantation; retinal ganglion cell; glaucoma; regeneration; neuroprotection
TV Johnson, ND Bull and KR Martin

\section{Introduction}

Inner retinal diseases pose a major threat to vision throughout the world. Glaucoma, the most common neurodegenerative condition of the inner retina, is estimated to affect over 60 million people globally and to cause bilateral blindness in more than $14 \%$ of them. ${ }^{1}$ Reducing intraocular pressure (IOP) is the only clinically proven method of slowing the progression of glaucoma, ${ }^{2}$ but ocular hypotensive therapy for this disease is insufficient in some patients as it fails to halt visual deterioration. For these patients, neuroprotective treatments that can support surviving retinal ganglion cells (RGCs) might offer a new avenue to preserve vision. However, due to the insidious character of glaucomatous onset and progression, many affected patients experience significant vision loss even before diagnosis and treatment can occur. Therefore, novel therapies that are capable of restoring vision are also of interest. Indeed, glaucoma may not be the only inner retinal condition that would benefit from therapeutic approaches that address these issues. It is possible that the findings discussed in this review may be applicable to other diseases that result in the death or dysfunction of RGCs, such as ischaemic optic neuropathy, optic neuritis, or the inherited mitochondrial optic neuropathies.

Transplantation of stem and progenitor cells is of great interest in the context of treating neurodegenerative disease in general, and in the retina in particular, because it may confer therapeutic benefit through both neuroprotective and cell replacement mechanisms. ${ }^{3-6}$ In the outer retina, the regenerative potential of cell transplantation has already been shown. Subretinal injection of photoreceptor progenitors obtained from mice at an optimal developmental stage permitted photoreceptor differentiation, integration of grafted cells, and functional recovery in rhodopsin-deficient host mice. ${ }^{7,8}$ Of course, the targeted replacement of RGCs is a more 
complex task than the replacement of photoreceptors, as RGC regeneration requires differentiation into a cell type with highly specialized properties, the establishment of numerous synaptic inputs, and the extension of an extremely long axon to precise brain targets in a manner that preserves the retinotopic map.

The prerequisites for successful cell transplantation to achieve neuroprotective goals are more modest and consist mainly of translocation into retinal tissue, long-term survival, and the provision of neurotrophic support for the host tissue. Indeed, some reports suggest that intraocular cell transplantation could benefit a variety of neurodegenerative conditions of the inner and outer retina by providing trophic support to surviving tissue $^{9-12}$ or stimulating endogenous support mechanisms. ${ }^{13,14}$ However, outstanding problems remain before inner retinal cell therapy in either context (regenerative or neuroprotective) will be a feasible clinical treatment option-What types of cells should be transplanted? Is transplantation into the eye sufficient to achieve an optimal therapeutic effect? What can be done to improve the efficacy of stem cell therapy for the inner retina? Here, we examine some of the research from our laboratory, which addresses the prospects for cell transplantation to treat diseases of the inner retina.

\section{Transplantation of MIO-M1 stem cells}

In 2002, Limb et al ${ }^{15}$ described a spontaneously immortalized cell line derived from the Müller glia of a postmortem human retina, which they designated MIO-M1. More recently, they showed that this and other similar cell lines are multipotent and capable of differentiating into neurons with a retinal phenotype. ${ }^{16}$ These data suggest that the adult human retina may harbour a population of cells that could be utilized for cell therapy. As such, we sought to study the behaviour of these cells after transplantation into glaucomatous rat eyes.

We found that xenotransplantation into either the vitreous or subretinal space without immune suppression resulted in the rapid rejection of grafted cells. In contrast, subsequent triple immunosuppressive therapy greatly enhanced cell survival. ${ }^{17}$ Histology revealed that grafted cells tended to remain as a bolus at the site of injection without any substantial integration into the host neural tissue (Figure 1a). However, intraocular administration of erythropoietin or chondroitinase ABC modestly increased the number of cells able to migrate into the retinal tissue (Figure 1b). In addition, although integrated cells extended processes into adjacent layers of the retina, they failed to exhibit any markers of differentiation. Occasionally, intravitreally engrafted cells expressed the neuronal marker $\beta$-III tubulin or the astrocytic marker GFAP, but robust differentiation of the transplant was not observed. These results are an encouraging demonstration that intraocular cell transplantation is feasible in a rat model of glaucoma, but also indicate that manipulation of the cells and/or host environment before transplantation will be necessary to optimize therapeutic results.

\section{Transplantation of oligodendrocyte precursor cells (OPCs)}

Having developed a reliable in vivo system with which to study retinal cell therapy, we opted to expand the pool of our graft sources to include OPCs isolated from the neonatal rat brain. OPCs have previously been shown to myelinate RGC axons when transplanted into the rat eye concomitant with an 'activating' inflammatory trigger. ${ }^{18}$ OPCs have also previously shown a neuroprotective capacity in vitro through neurotrophic factor secretion. ${ }^{19}$ Thus, we were interested to know whether either of these phenomena would confer neuroprotection to RGCs upon OPC transplantation into glaucomatous eyes.

In this case, syngeneic transplants using inbred Lewis rats were utilized in the absence of immune suppression to limit graft rejection and avoid potential complication introduced by administration of immunosuppressive drugs. Syngeneic transplantation resulted in graft survival through the 12-week duration of our study. Transplantation of OPCs alone, either 8 weeks before or concurrent with the onset of glaucoma, did not offer any neuroprotective effect. However, coinjection of OPCs and an inflammatory stimulus increased axonal survival by approximately 50 percent. $^{20}$ Moreover, we observed that the engrafted cells differentiated into mature oligodendrocytes (as expected) and produced cells that expressed GFAP or $\beta$-III tubulin, suggestive of astrocytic or neuronal differentiation, respectively. Interestingly, limited myelination of RGC axons by differentiating OPCs was observed which hinted that the neuroprotective effect was not contact-mediated, but due to the supply of soluble neurotrophic factors. Future research will address the neuroprotective mechanism of OPC transplantation and the potential for OPCs to be used in a regenerative capacity.

\section{A new in vitro model of cell transplantation for the inner retina}

The results of our in vivo experiments and that of other laboratories have provided encouraging proof of principle that cell transplantation can have beneficial 
effects on neurodegenerative conditions of the inner retina. However, they also show the limitations of intraocular cell transplantation. When we transplanted
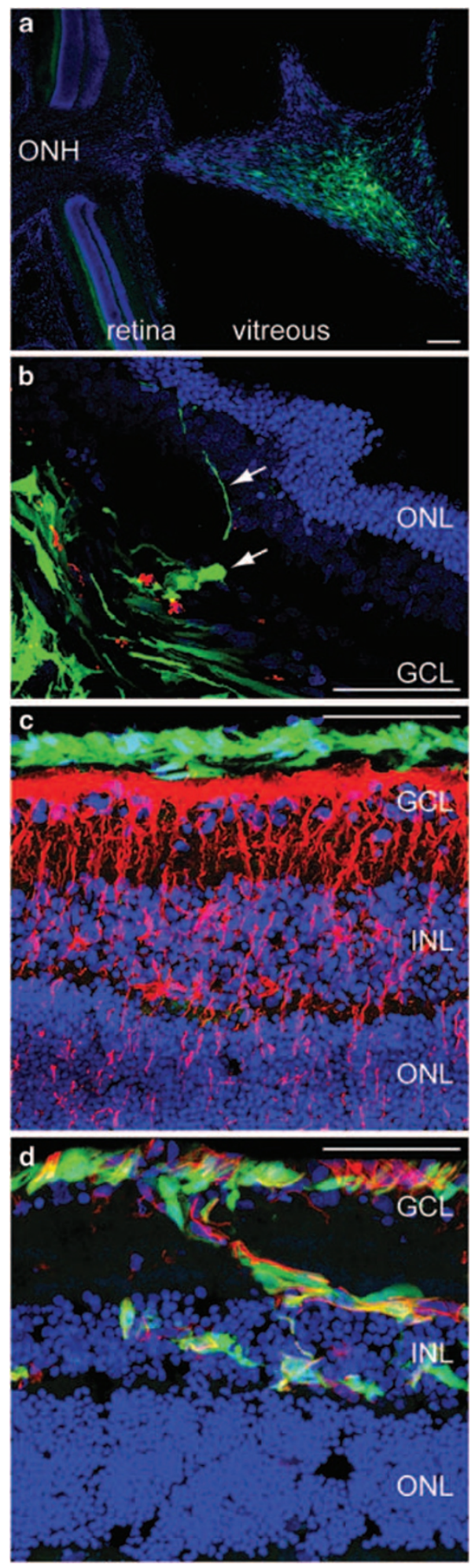

MIO-M1 stem cells or OPCs, we observed minimal retinal integration $(<1 \%$ of cells) and could not adequately control or encourage the differentiation of engrafted cells. It was also evident that using an in vivo approach to improve the efficacy of intraocular transplantation was problematic as we encountered graft rejection, needed to utilize a large number of animals to obtain statistically significant results, and were unable to control the host environment after cell transplantation. Thus, we developed and characterized an in vitro system with which to efficiently screen compounds and techniques that might enhance the efficacy of stem cell therapy. ${ }^{21}$

Using a serum-free media formulation, we developed a system whereby organotypic retinal explants from adult rats could be cultured for up to 17 days. We showed that tissue survival was good, as evidenced by prolonged cell survival, propidium iodide exclusion, low cleaved-caspase-3 labelling, and sustained immunoreactivity for a panel of neural retinal proteins. Importantly, retinal explants responded to coculture of either MIO-M1 cells or bone marrow-derived mesenchymal stromal cells (MSCs) on the vitreal surface in a manner very similar to whole eyes that received intravitreal cell injections. Endogenous Müller glia upregulated reactive intermediate filaments and the integration of grafted or co-cultured cells was blocked at the inner limiting membrane (ILM, Figure 1c). Therefore, we concluded that this model could be used to screen interventions to enhance retinal integration of transplanted cells rapidly while utilizing only $1 / 8$ the number of animals used for comparative in vivo experiments.

Figure 1 The retina is resistant to transplanted stem cell migration, and environmental modulation can enhance integration. (a) MIO-M1 stem cells (green) transplanted in vivo into the vitreous remained as a bolus and were unable to migrate into the glaucomatous rat retina. Nuclei were counterstained with DAPI (blue). (b) Treatment of the retina with either erythropoietin or chondroitinase ABC permitted some engrafted MIO-M1 cells (green) to migrate into the injured retina and extend processes across several retinal layers (arrows). Immune cells are labelled with ED1 (red). Nuclei were counterstained with DAPI (blue). (c) Transplantation of bone marrow-derived mesenchymal stromal cells (MSCs, green) onto the in vitro retinal explant vitreal surface was associated with the upregulation of glial reactivity markers, such as nestin (red) in Müller cells. Nuclei were counterstained with DAPI. (d) Treatment of retinal explants with a selective glial toxin resulted in glial reactivity blockade and permitted the migration of grafted cells into the retinal tissue. Nuclei were counterstained with DAPI in blue. $\mathrm{ONH}=$ optic nerve head; $\mathrm{GCL}=$ ganglion cell layer; $\mathrm{INL}=$ inner nuclear layer; $\mathrm{ONL}=$ outer nuclear layer; scale bar $=100 \mu \mathrm{m}$. 


\section{Identifying and overcoming obstacles to intravitreal cell transplantation}

Throughout our experiments, we have consistently observed that intravitreally transplanted cells can migrate into very close proximity with the inner retinal surface but rarely progress past the ILM. Having made a similar observation in retinal explant cultures, we used this system to identify the specific barrier obstructing grafted-cell integration. Initially, we mechanically peeled the ILM from the explant vitreal surface and confirmed immunohistochemically that the inner basal lamina was removed from portions of the tissue along with, in some cases, Müller cell endfeet. Importantly, ILM peeling before stem cell transplantation onto the vitreal surface resulted in a dramatic increase in the migration of engrafted cells into the retina, indicating that a major obstacle to cell migration exists within the ILM. We were curious, however, whether the specific barrier resided at the level of the basement membrane or at the glial endfeet. By independently disrupting each of these factors in isolation, using either enzymatic protein digestion or glial-specific toxins respectively, we determined that the integrity of the inner basal lamina is neither necessary nor sufficient to prevent grafted-cell infiltration into the retina. In contrast, suppression of Müller cell reactivity dramatically enhanced graft integration (Figure 1d). It should be noted that the development of our in vitro retinal explant system was essential to show that basal lamina digestion did not enhance retinal graft migration because, unlike for the avascular chick retina, ${ }^{22,23}$ intravitreal injection of the proteolytic enzyme collagenase into rat eyes caused acute retinal haemorrhage, thereby severely limiting investigations in vivo.

\section{Future directions}

Although progress has been made in developing stem cell-based therapies for inner retinal disease, many barriers to clinical efficacy remain. Identifying ethical and clinically available sources for cells will require careful consideration. Although embryonic- and foetalderived stem cells have shown the capacity to generate retinal-like neurons ${ }^{24-28}$ and confer retinal neuroprotection, ${ }^{12,29}$ ethical and logistic issues, including graft rejection resulting from inherent allogenic transplantation, will likely restrict their clinical utility. Various types of stem/progenitor cells exist in the adult mammalian body, including the eye ${ }^{30-33}$ and further research into using these cells for transplantation is of interest. A newly revealed prospect exists in the use of induced pluripotent stem (iPS) cells, which can be produced from postmitotic adult tissue, such as skin, to create cells with properties similar to ES cells. ${ }^{34,35}$ Whether these cells will be useful for retinal stem cell therapy remains unknown. Regardless of the cell type chosen for transplantation, methods to safely control the terminal differentiation (for cell replacement) or bioactive factor secretion (for neuroprotection) must be further developed.

Although we have discussed methods to enhance the migration of transplanted cells into retinal tissue, further work is necessary. Modulation of the retinal environment to enhance graft integration should be optimized while minimizing disruption of normal retinal function. Furthermore, once engrafted cells can structurally integrate into the retina, they must then extend neurites and make synaptic connections with neighbouring cells. This will likely require the modulation of the notoriously inhibitory CNS environment. Treatment with chondroitinase $\mathrm{ABC}$, for instance, digests chondroitin sulphate proteoglycans and enhances neurite outgrowth in the spinal cord ${ }^{36}$ and in the retina. ${ }^{17,37}$ Combination treatments that promote migration, neurite growth, and synaptogenesis may be required for the full integration of transplanted cells. Thus, although formidable obstacles remain before cell transplantation will be used clinically to treat inner retinal disease, stepwise progress is leading us closer to protocols that may eventually demonstrate efficacy.

\section{Acknowledgements}

This study was supported by a GSK Clinician Scientist Fellowship (KRM), the Glaucoma Research Foundation, Addenbrooke's Hospital (Cambridge, UK), a GatesCambridge Scholarship (TVJ), an NIH-Graduate Partnership Programme Fellowship (TVJ), and the Richard Norden Glaucoma Research Fund.

\section{References}

1 Quigley HA, Broman AT. The number of people with glaucoma worldwide in 2010 and 2020. Br J Ophthalmol 2006; 90: 262-267.

2 Kass MA, Heuer DK, Higginbotham EJ, Johnson CA, Keltner JL, Miller JP et al. The Ocular Hypertension Treatment Study: a randomized trial determines that topical ocular hypotensive medication delays or prevents the onset of primary open-angle glaucoma. Arch Ophthalmol 2002; 120: 701-713; discussion 829-730.

3 Levin LA, Ritch R, Richards JE, Borras T. Stem cell therapy for ocular disorders. Arch ophthalmol 2004; 122: 621-627.

4 Quigley HA, Iglesia DS. Stem cells to replace the optic nerve. Eye 2004; 18: 1085-1088.

5 Bull ND, Martin KR. Optic nerve restoration: new perspectives. J Glaucoma 2007; 16: 506-511. 
6 Bull ND, Johnson TV, Martin KR. Stem cells for neuroprotection in glaucoma. Prog Brain Res 2008; 173: 511-519.

7 MacLaren RE, Pearson RA, MacNeil A, Douglas RH, Salt $\mathrm{TE}$, Akimoto $\mathrm{M}$ et al. Retinal repair by transplantation of photoreceptor precursors. Nature 2006; 444: 203-207.

8 Bartsch U, Oriyakhel W, Kenna PF, Linke S, Richard G, Petrowitz B et al. Retinal cells integrate into the outer nuclear layer and differentiate into mature photoreceptors after subretinal transplantation into adult mice. Exp Eye Res 2008; 86: 691-700.

9 Inoue Y, Iriyama A, Ueno S, Takahashi H, Kondo M, Tamaki $\mathrm{Y}$ et al. Subretinal transplantation of bone marrow mesenchymal stem cells delays retinal degeneration in the RCS rat model of retinal degeneration. Exp eye res 2007; 85: 234-241.

10 Gamm DM, Wang S, Lu B, Girman S, Holmes T, Bischoff N et al. Protection of visual functions by human neural progenitors in a rat model of retinal disease. PLOS ONE 2007; 2: e338.

11 Schraermeyer U, Thumann G, Luther T, Kociok N, Armhold $\mathrm{S}$, Kruttwig K et al. Subretinally transplanted embryonic stem cells rescue photoreceptor cells from degeneration in the RCS rats. Cell Transplant 2001; 10: 673-680.

12 Meyer JS, Katz ML, Maruniak JA, Kirk MD. Embryonic stem cell-derived neural progenitors incorporate into degenerating retina and enhance survival of host photoreceptors. Stem Cells 2006; 24: 274-283.

13 Charalambous P, Hurst LA, Thanos S. Engrafted chicken neural tube-derived stem cells support the innate propensity for axonal regeneration within the rat optic nerve. Invest Ophthalmol Vis Sci 2008; 49: 3513-3524.

14 Zhang Y, Klassen HJ, Tucker BA, Perez MT, Young MJ. CNS progenitor cells promote a permissive environment for neurite outgrowth via a matrix metalloproteinase-2dependent mechanism. J Neurosci 2007; 27: 4499-4506.

15 Limb GA, Salt TE, Munro PM, Moss SE, Khaw PT. In vitro characterization of a spontaneously immortalized human Muller cell line (MIO-M1). Invest Ophthalmol Vis Sci 2002; 43: 864-869.

16 Lawrence JM, Singhal S, Bhatia B, Keegan DJ, Reh TA, Luthert PJ et al. MIO-M1 cells and similar muller glial cell lines derived from adult human retina exhibit neural stem cell characteristics. Stem Cells 2007; 25: 2033-2043.

17 Bull ND, Limb GA, Martin KR. Human Muller stem cell (MIO-M1) transplantation in a rat model of glaucoma: survival, differentiation, and integration. Invest Ophthalmol Vis Sci 2008; 49: 3449-3456.

18 Setzu A, Lathia JD, Zhao C, Wells K, Rao MS, Ffrench-Constant $\mathrm{C}$ et al. Inflammation stimulates myelination by transplanted oligodendrocyte precursor cells. Glia 2006; 54: 297-303.

19 Wilkins A, Chandran S, Compston A. A role for oligodendrocyte-derived IGF-1 in trophic support of cortical neurons. Glia 2001; 36: 48-57.

20 Bull ND, Irvine KA, Franklin RJM, Martin KR. Intravitreal Transplantation of Oligodendrocyte Precursor Cells Increases Retinal Ganglion Cell Survival in a Rat Glaucoma Model. Invest Ophthalmol Vis Sci 2008; 49; ARVO E (abstract 4064).

21 Johnson TV, Martin KR. Development and characterization of an adult retinal explant organotypic tissue culture system as an in vitro intraocular stem cell transplantation model. Invest Ophthalmol Vis Sci 2008; 49: 3503-3512.

22 Halfter W, Willem M, Mayer U. Basement membranedependent survival of retinal ganglion cells. Invest Ophthalmol Vis Sci 2005; 46: 1000-1009.

23 Halfter W, Winzen U, Bishop PN, Eller A. Regulation of eye size by the retinal basement membrane and vitreous body. Invest Ophthalmol Vis Sci 2006; 47: 3586-3594.

24 Banin E, Obolensky A, Idelson M, Hemo I, Reinhardtz E, Pikarsky E et al. Retinal incorporation and differentiation of neural precursors derived from human embryonic stem cells. Stem Cells 2006; 24: 246-257.

25 Ikeda H, Osakada F, Watanabe K, Mizuseki K, Haraguchi T, Miyoshi $\mathrm{H}$ et al. Generation of $\mathrm{Rx}+/ \mathrm{Pax} 6+$ neural retinal precursors from embryonic stem cells. Proc Natl Acad Sci USA 2005; 102: 11331-11336.

26 Lamba DA, Karl MO, Ware CB, Reh TA. Efficient generation of retinal progenitor cells from human embryonic stem cells. Proc Natl Acad Sci USA 2006; 103: 12769-12774.

27 Tabata Y, Ouchi Y, Kamiya H, Manabe T, Arai K, Watanabe S. Specification of the retinal fate of mouse embryonic stem cells by ectopic expression of Rx/rax, a homeobox gene. Mol Cell Biol 2004; 24: 4513-4521.

28 Osakada F, Ikeda H, Mandai M, Wataya T, Watanabe K, Yoshimura $\mathrm{N}$ et al. Toward the generation of rod and cone photoreceptors from mouse, monkey and human embryonic stem cells. Nat biotechnol 2008; 26: 215-224.

29 Lund RD, Wang S, Klimanskaya I, Holmes T, Ramos-Kelsey $\mathrm{R}, \mathrm{Lu} \mathrm{B}$ et al. Human embryonic stem cell-derived cells rescue visual function in dystrophic RCS rats. Cloning Stem Cells 2006; 8: 189-199.

30 Ahmad I, Tang L, Pham H. Identification of neural progenitors in the adult mammalian eye. Biochem Biophys Res Commun 2000; 270: 517-521.

31 Haruta M, Kosaka M, Kanegae Y, Saito I, Inoue T, Kageyama R et al. Induction of photoreceptor-specific phenotypes in adult mammalian iris tissue. Nat Neurosci 2001; 4: 1163-1164.

$32 \mathrm{Xu} \mathrm{H}$, Sta Iglesia DD, Kielczewski JL, Valenta DF, Pease ME, Zack DJ et al. Characteristics of progenitor cells derived from adult ciliary body in mouse, rat, and human eyes. Invest ophthalmol vis sci 2007; 48: 1674-1682.

33 Mayer EJ, Carter DA, Ren Y, Hughes EH, Rice CM, Halfpenny CA et al. Neural progenitor cells from postmortem adult human retina. Br j ophthalmol 2005; 89: 102-106.

34 Yu J, Vodyanik MA, Smuga-Otto K, Antosiewicz-Bourget J, Frane JL, Tian $\mathrm{S}$ et al. Induced pluripotent stem cell lines derived from human somatic cells. Science 2007; 318 : 1917-1920.

35 Takahashi K, Tanabe K, Ohnuki M, Narita M, Ichisaka T, Tomoda $\mathrm{K}$ et al. Induction of pluripotent stem cells from adult human fibroblasts by defined factors. Cell 2007; 131: 861-872.

36 Bradbury EJ, Moon LD, Popat RJ, King VR, Bennett GS, Patel PN et al. Chondroitinase ABC promotes functional recovery after spinal cord injury. Nature 2002; 416: 636-640.

37 Singhal S, Lawrence JM, Bhatia B, Ellis JS, Kwan AS, Macneil A et al. Chondroitin sulfate proteoglycans and microglia prevent migration and integration of grafted Muller stem cells into degenerating retina. Stem Cells 2008; 26: 1074-1082. 